\title{
The role of Ar III in quantitative spectroscopy on hot argon plasmas
}

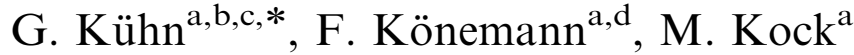

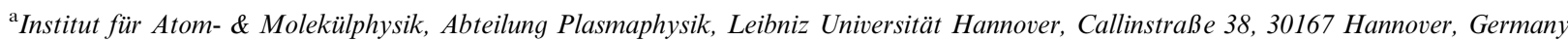 \\ ${ }^{\mathrm{b}}$ Institut für Gravitationsphysik, Leibniz Universität Hannover, Callinstraße 38, 30167 Hannover, Germany \\ ${ }^{\mathrm{c}}$ Max-Planck-Institut für Gravitationsphysik, Albert-Einstein-Institut, Callinstraße 38, 30167 Hannover, Germany \\ ${ }^{\mathrm{d}}$ Inside M2M GmbH, Schönebecker Allee 2, 30823 Garbsen (Hannover), Germany
}

Received 26 April 2006; received in revised form 21 September 2006; accepted 22 September 2006

\begin{abstract}
We perform quantitative optical emission spectroscopy on the hot core of the cathode region of a free-burning arc in argon under atmospheric pressure. As the peak temperatures in the centre of the discharge exceed $22000 \mathrm{~K}$ we are able to observe three spectra of argon (Ar I, Ar II and Ar III) and the continuum emission. We report on some inconsistencies concerning the evaluation of Ar III radiation in both line and continuum emission. These are caused by erroneous data in the literature and common misconceptions about the influence of Ar III on the plasma emission. We discuss the impact of this fact on published data.
\end{abstract}

(C) 2006 Elsevier Ltd. All rights reserved.

PACS: 52.25.-b; 52.25.Jm; 52.70.-m; 52.80.Mg

Keywords: Ar III; Partition function; $\xi$-factor; Line emission; Continuum emission

\section{Introduction}

In previous papers $[1,2]$ we presented results from diagnostics in the near-cathode region of a low-current free-burning arc in argon under atmospheric pressure. Our main tool of investigation was optical emission spectroscopy. We observed a discharge mode called "blue core mode" which featured a hot plasma region in front of the cathode with electron temperatures exceeding $22000 \mathrm{~K}$. These temperatures allowed us to observe three spectra of argon (Ar I, Ar II and Ar III) and the continuum emission. We found that the plasma was, in spite of the high temperatures and high electron densities, not in the state of local thermodynamic equilibrium (LTE) due to the vicinity of the cathode, but could be described by means of a model for partial thermodynamic equilibrium (pLTE) (see $[2,3]$ ).

\footnotetext{
*Corresponding author. Institut für Atom- \& Molekülphysik, Abteilung Plasmaphysik, Leibniz Universität Hannover, Callinstraße 38, 30167 Hannover, Germany

E-mail address: gerrit@pmp.uni-hannover.de (G. Kühn).
} 
For the evaluation of emission spectroscopical data we needed corresponding calculations for the LTE and the pLTE plasma model. In doing so we came across some peculiarities connected with the contribution of Ar III to the plasma emission.

The details of the experimental setup and the evaluation of the data can be found in [2-5] and are not relevant for the following considerations. Here we give a short summary of both. We observed the emission of a free-burning arc side-on. The established spectroscopical $\mathrm{T}$-arrangement allowed us to measure radiation on an absolute scale. A mirror-tower rotated the (live-sized) image of the discharge by $90^{\circ}$, so that radial side-on measurements could be performed along the entrance slit of a monochromator. The data were Abel inverted with the inversion algorithm described in [6] to yield spatially resolved emission coefficients for argon line and continuum emission. The radial resolution was defined by the pixel-size of the CCD detector used $(\approx 30 \mu \mathrm{m})$. The axial resolution was defined by the width of the entrance slit, which we set to $50 \mu \mathrm{m}$. We selected different axial sheaths with a DC motor connected to one of the mirrors. The cathode used was typically a cylindrical rod of thoriated tungsten $(1.8 \% \mathrm{Th})$ with a diameter of $0.6 \mathrm{~mm}$ having flat ends.

In $[1,2]$ we found large deviations from LTE in the vicinity of the cathode in spite of high temperatures above $20000 \mathrm{~K}$ and high electron densities up to $2 \times 10^{23} \mathrm{~m}^{-3}$. Other authors report similar findings on highcurrent arcs [7-11]. In this paper we analyze the evaluation procedure of the spectroscopic data to check that the measured values for $n_{\mathrm{e}}$ and $T_{\mathrm{e}}$ (and thus the deviations from the LTE state) are free from systematic errors.

The basis for the description of high-pressure arc plasmas is the concept of LTE. Our procedure to calculate LTE plasma compositions was an extension of the approach published in [12,13] and included Dalton's law, the quasi-neutrality condition and Saha's equation (see [3] for a more detailed description of the mathematical procedure). Additionally the Boltzmann distribution was used to evaluate argon line emission.

Our calculations included ionization stages up to $i=4$ viz. Ar V. In practice (and especially for evaluation of measured spectroscopic data) calculations are often limited to stages up to $i=1$ (singly ionized plasmas). This simplifies the equations, because one can set $n_{1}=n_{\mathrm{e}}$. This assumption is justified as long as the difference between $n_{1}$ and $n_{\mathrm{e}}$ (and thus the influence of ArIII) on the plasma composition can be neglected.

\section{The role of Ar III}

\subsection{Calculation of partition functions}

For plasma model calculations as well as for the evaluation of spectroscopic data we have to calculate the atomic partition functions $Z_{i}$ of the various ionization stages of argon, which enter the Saha equation and the Boltzmann distribution. The partition functions can be described as weighted sum over all energy levels:

$$
Z_{i}:=\sum_{v=0}^{v_{\max }}\left[g_{i}|v\rangle \exp \left(-\frac{E_{i}^{|v\rangle}}{k_{\mathrm{B}} T}\right)\right], \quad \text { with } E_{i}^{\left|v_{\max }\right\rangle} \leqslant\left(E_{i}^{\infty}-\Delta E_{i}^{\infty}\right) .
$$

Here $i$ denotes the degree of ionization, $E^{|v\rangle}$ is the excitation energy of the level $|v\rangle, g^{|v\rangle}$ is the corresponding statistical weight, and $E_{i}^{\infty}$ is the ionization energy. $\Delta E_{i}^{\infty}$ is the depression of the ionization energy according to [14], and $v_{\max }$ denotes the resulting highest energy level included into the summation. The energy depression ensures that the sum does not diverge.

On a first sight the calculation of $Z_{i}$ appears to be a straightforward process that can be achieved by explicitly executing the sum in Eq. (1). But this is only possible if the contributing energy levels are known. However, especially at higher temperatures many of the excited states have to be known and summed up to yield a good value for the partition function.

We calculate partition functions approximately by applying methods given in [15] (other interesting sources of information about the calculation of partition functions are, e.g. [16-18]). Thereby the sum in Eq. (1) is split into three parts:

(1) the term for the ground state $|0\rangle$,

(2) a sum containing (known) energetically low-lying levels, and

(3) a sum over the higher excited levels up to the reduced ionization limit. 


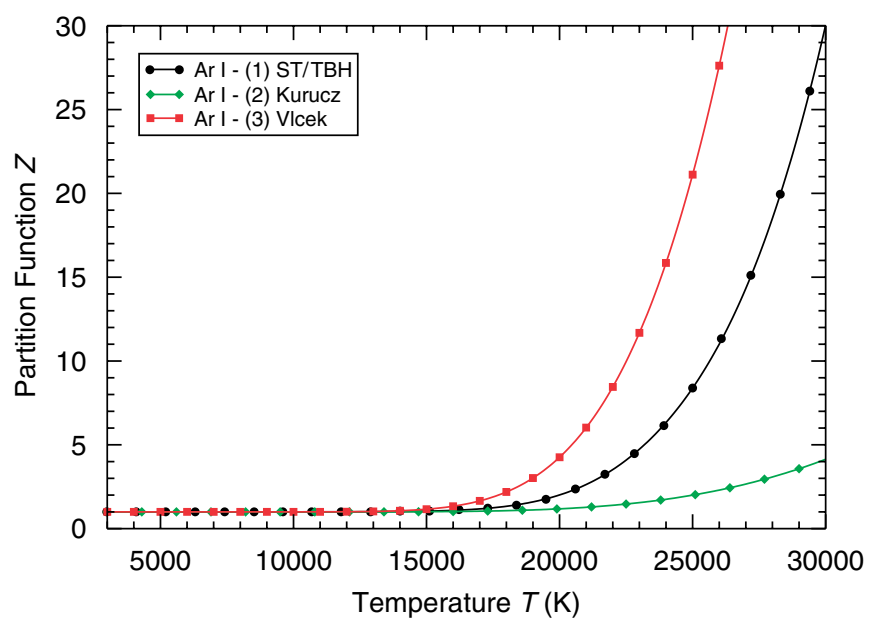

Fig. 1. Comparison of different methods for calculating the partition function for Ar I: (1) according to [15,19], (2) using data from [20], and (3) using data from [21] (see text for details).

The first part is easy to evaluate as it consists only of the statistical weight $g_{i}^{|0\rangle}$. The second part is substituted by a few equivalent levels and statistical weights, which are the result of a Čebyšev approximation. The coefficients are taken from [19]. Thirdly the highly excited terms are treated as hydrogen-like and can thus be calculated elementary. This method has various advantages: it requires only little input data, reduces the computation time and simultaneously incorporates the often not well-known highly excited states.

Fig. 1 shows the result for three different ways of calculating the partition function of Ar I:

(1) the method of $[15,19]$, as described above,

(2) an explicit summation over known energy terms (taken from [20]),

(3) an explicit summation over 65 artificial energy levels originally created for a collisional-radiative model in [21].

The partition functions in Fig. 1 diverge at temperatures above $15000 \mathrm{~K}$. Procedure (2) certainly underestimates the true value for the partition function because of missing states. On the other hand, procedure (3) gives noticeably higher values already around $15000 \mathrm{~K}$. In our opinion the highly excited artificial energy levels in [21] (with statistical weights up to $g_{0}^{|65\rangle}=2888$ ) are overestimated, and, therefore, we believe that the results of procedure (1), which lie between (2) and (3), represent the best available approximation.

However, when we re-analyzed our evaluation procedure for the spectroscopic data to ensure that the deviations from LTE reported in [2] are real and the measured values for $n_{\mathrm{e}}$ and $T_{\mathrm{e}}$ are reliable, we found a discrepancy in the partition function of Ar III. Fig. 2 compares the results we obtained with the method of $[15,19]$ with the results from a direct summation over the Ar III terms published in [22].

There is an obvious difference in the calculated partition functions already at $5000 \mathrm{~K}$ (see Fig. 2) which we cannot explain by a different treatment of the hydrogenic part of the partition function. Therefore, we suppose that the Cebyšev coefficients for Ar III published in [19] are questionable and that the direct summation gives better results in this case. The impact of the partition function of Ar III on the plasma composition is shown in Fig. 3. The different densities $n_{2}$ and (for higher temperatures) also $n_{0}$ and $n_{1}$ are obvious, even on a logarithmic scale. On the other hand, the electron density $n_{\mathrm{e}}$ remains effectively the same.

It is hard for us to estimate how many publications are affected by this error, because unfortunately many authors do not mention the calculation method they use for partition functions. However, as the method of $[15,19]$ is recommended in standard books on plasma spectroscopy like [23], we suppose that it is rather widespread. In the following section we will discuss (among other things) the influence of the Ar III partition 


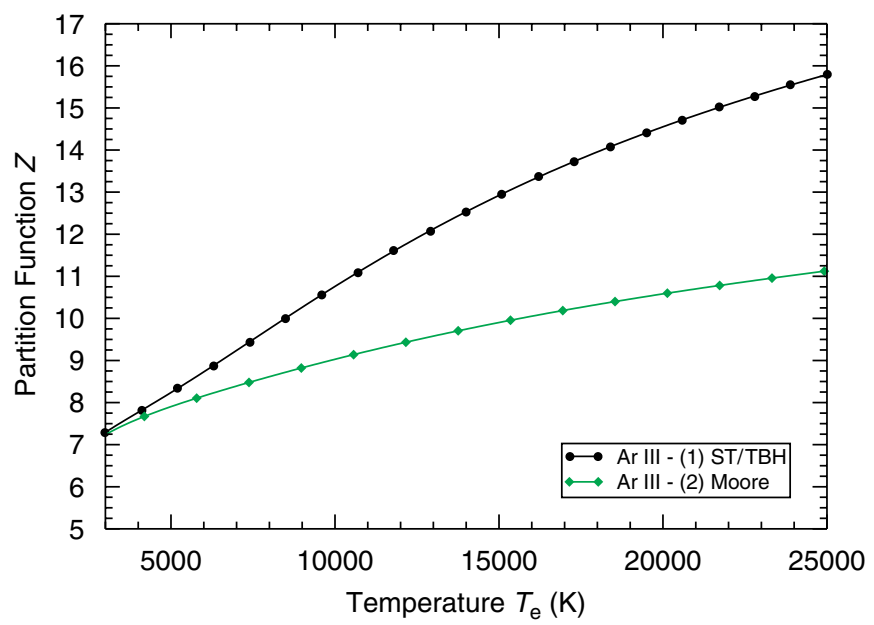

Fig. 2. Comparison of different methods for calculating the partition function for Ar III: (1) according to [15,19], (2) using data from [22] (see text for details).

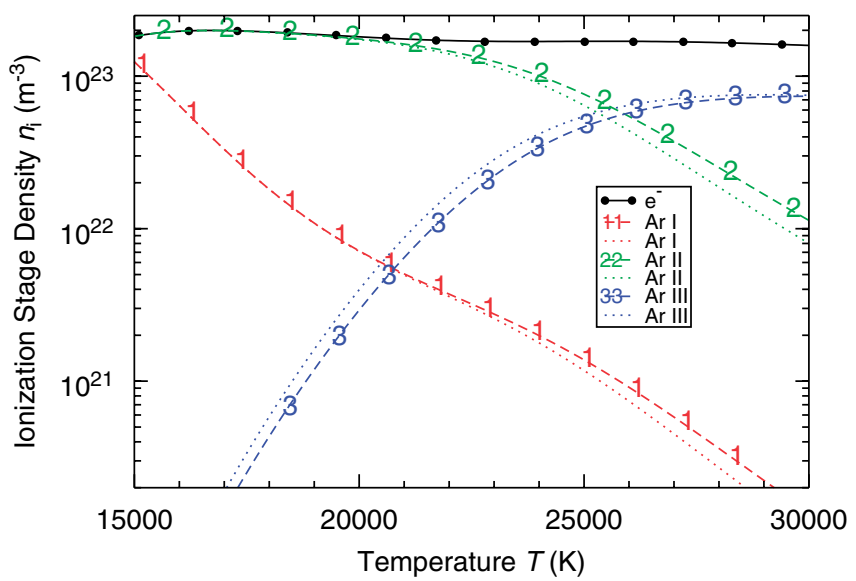

Fig. 3. LTE calculation of electron and ionization stage densities using different approaches for the partition function of Ar III. The broken curves use method (2) from Fig. 2, the dotted curves use method (1).

function on spectroscopically determined plasma parameters using our measurements in the cathode region of our free-burning arc discharge as an example.

\subsection{Ar III in line emission (determination of $T_{\mathrm{e}}$ )}

Fig. 4 shows an LTE calculation for the line emission coefficient (LEC) for the first three ionization stages of argon, each stage is represented by two emission lines. The measurement of an absolute LEC is a common method for determining the excitation temperature of a plasma species (see e.g. [3,23]).

With our experimental setup the usage of Ar III lines to determine the temperature is feasible from about $19000 \mathrm{~K}$ on. The usage of the next higher ionization species is especially advisable when the temperature exceeds the so-called norm temperature. As noted in Fig. 4 the norm temperature for ArI lines is about $15000 \mathrm{~K}$ and the norm temperature for Ar II lines is $26000 \mathrm{~K}$ (Table 1). Temperature determination above the norm temperature is no longer unambiguous and becomes more difficult, because the LEC reaches the socalled Larenz maximum [24] and declines for higher temperatures. For free-burning arcs we had to perform 


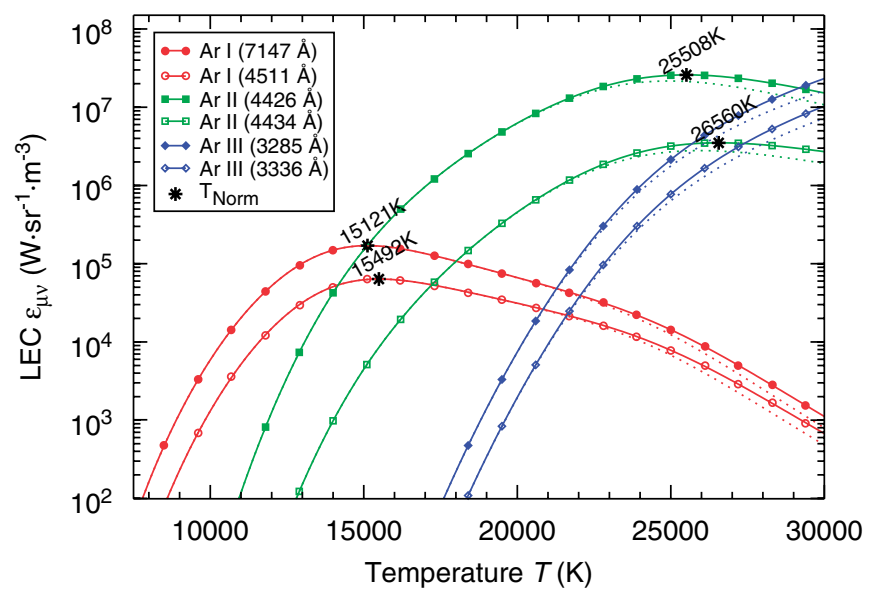

Fig. 4. LTE calculation of line emission coefficients for different argon lines using different approaches for the partition function of Ar III. The solid curves use method (2) from Fig. 2, the dotted curves use method (1). The atomic data used for the calculations can be found in Table 1. The maximum of each curve (marked by an asterisk) is the Larenz maximum, the corresponding temperature is the norm temperature.

Table 1

Atomic data of the transitions used in this paper

\begin{tabular}{|c|c|c|c|c|c|c|}
\hline $\mathrm{S}$. & $\lambda_{\mu v}(\AA)$ & $E_{\mu}\left(\mathrm{cm}^{-1}\right)$ & $g_{\mu}$ & $A_{\mu v}\left(10^{6} \mathrm{~Hz}\right)$ & U. (\%) & Ref. \\
\hline ArI & 4510.73 & 117563 & 1 & 1.18 & 7 & [26] \\
\hline ArI & 7147.04 & 107132 & 3 & 0.625 & 8 & [26] \\
\hline Ar II & 4426.0 & 157673 & 6 & 81.7 & 5 & [27] \\
\hline Ar II & 4433.84 & 194883 & 8 & 64.98 & 10 & [28] \\
\hline Ar III & 3285.85 & 204797 & 7 & 200.0 & 10 & [29] \\
\hline Ar III & 3336.13 & 226646 & 9 & 200.0 & 10 & [29] \\
\hline
\end{tabular}

Given are the species type (S.), the transition wavelength $\left(\lambda_{\mu v}\right)$, the excitation energy of the upper energy level $\left(E_{\mu}\right)$, the statistical weight $\left(g_{\mu}\right)$, the transition probability $\left(A_{\mu v}\right)$, the uncertainty for the A value (U.) and the reference (Ref.).

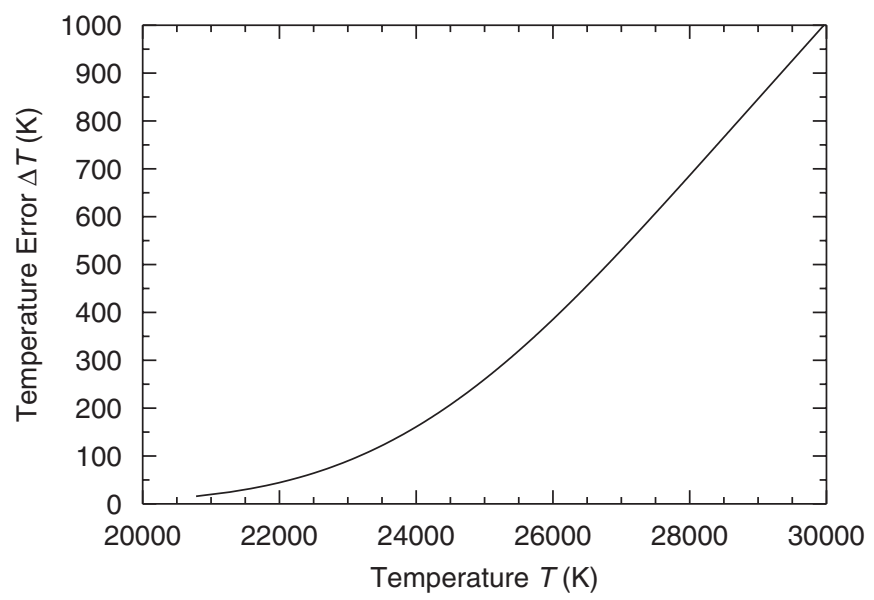

Fig. 5. LTE calculation of the temperature error resulting from using method (1) from Fig. 2 for the partition function for Ar III.

side-on measurements, which were integrated along the line-of-sight and had to be Abel inverted. However, the errors for an Abel inversion become significantly larger if the line shows an off-axis maximum [25] as is the case when observing an LEC over a temperature range which includes the norm temperature). 


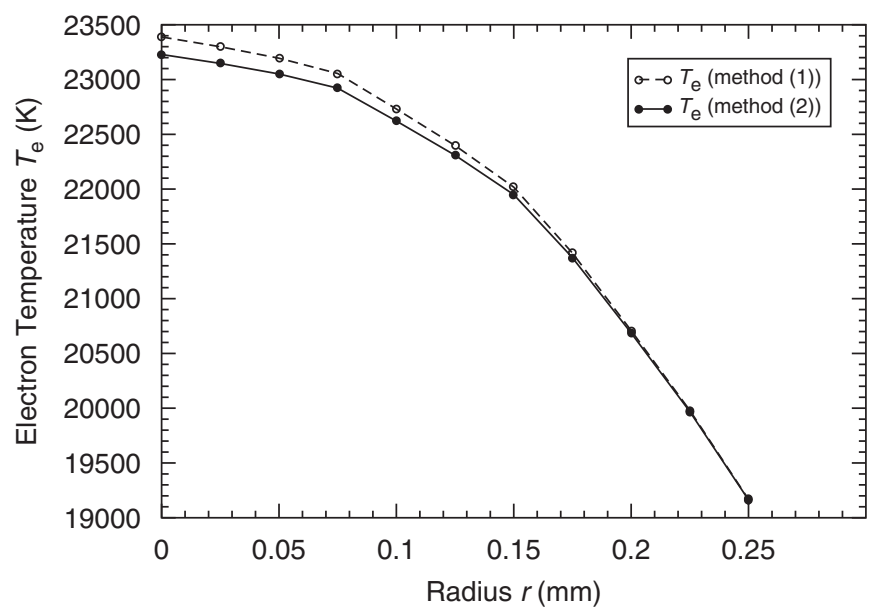

Fig. 6. Determination of $T_{\mathrm{e}}$ in the cathode region of our free-burning arc from Ar III line emission using different approaches for the partition function of Ar III. The solid curves use method (2) from Fig. 2, the broken curves employ method (1). The distance from the cathode was approximately $100 \mu \mathrm{m}$, the cathode diameter was $600 \mu \mathrm{m}$ and the arc current was $10 \mathrm{~A}$. The measured data were taken from [2]. $T_{\mathrm{e}}$ was calculated with a pLTE model using line emission at $3286 \AA$ and continuum emission (see $[2,3]$ ).

Fig. 5 shows the error in temperature determination which is caused by using the Ar III partition function according to $[15,19]$. In both Figs. 4 and 5 it can be seen that the error in the partition function becomes more relevant for higher temperatures. As the partition function appears in Saha's equation as well as in the Boltzmann distribution, the error can almost completely cancel out in the determination of the temperature. This is known to happen at lower temperatures according to [30] when the relevant species (Ar III) is a minority species. However, at higher temperatures the wrong partition function leads to a clearly visible systematic error in the determination of the excitation temperature.

In [2] we published radial temperature profiles for our free-burning arc which were obtained using absolute line emission from different argon species and continuum emission together with a pLTE model, because the plasma in the near-cathode region is not in LTE. As Ar III lines yielded the highest temperatures and since we used the method from [15,19] to calculate the partition function, the question arises whether this can explain the difference of about $2000 \mathrm{~K}$ between temperatures obtained from Ar III and Ar II line emission.

Fig. 6 shows the re-evaluated data from [2] using partition function (2) instead of (1) from Fig. 2 for Ar III, which yields more reliable values as explained above. As expected the largest difference appears in the centre of the arc where the highest temperatures are reached. However, even at a core temperature of about $23500 \mathrm{~K}$, the systematic error caused by the wrong partition function is less than $200 \mathrm{~K}$.

Another possible reason for the discrepancies is the uncertainty in the A values used for the diagnostic lines. In [2] we used the values published in [31] which have a rather large error of about 50\%. However, meanwhile [29] published exactly the same A values with a smaller uncertainty of $10 \%$, so that this can be ruled out as a reason for the differences in temperature, too. Therefore, our warning from [2] not to over-interpret the results is still valid.

\subsection{Ar III in continuum emission (determination of $n_{\mathrm{e}}$ )}

Another interesting plasma parameter, besides the plasma temperature, is the electron density $n_{\mathrm{e}}$. In the case of LTE $n_{\mathrm{e}}$ and $T_{\mathrm{e}}$ can be calculated if one of the two is known, but in pLTE they have to be determined separately (see [3]). In atmospheric argon arc plasmas a popular method to determine $n_{\mathrm{e}}$ is the absolute measurement of the continuum emission coefficient (CEC) $\varepsilon_{\lambda}$, which is related to $n_{\mathrm{e}}$ and $T_{\mathrm{e}}$ [32]:

$$
\begin{aligned}
& \varepsilon_{\lambda}=\frac{e^{6}}{12 c^{2} \varepsilon_{0}^{3} \sqrt{6 \pi^{5} m_{\mathrm{e}}^{3} k_{\mathrm{B}}}} \frac{n_{\mathrm{e}}}{\lambda^{2} \sqrt{T_{\mathrm{e}}}} \sum_{i=1}^{N_{\mathrm{Ar}}} n_{i} i^{2} \xi_{i}\left(\lambda, T_{\mathrm{e}}\right) \quad \text { with } \\
& \xi_{i}:=\left[1-\exp \left(-\frac{h c}{\lambda k_{\mathrm{B}} T_{\mathrm{e}}}\right)\right] \frac{\gamma_{i}}{Z_{i}\left(T_{\mathrm{e}}, n_{\mathrm{e}}\right)} \xi_{i}^{\mathrm{fb}}\left(\lambda, T_{\mathrm{e}}\right)+\exp \left(-\frac{h c}{\lambda k_{\mathrm{B}} T_{\mathrm{e}}}\right) \xi_{i}^{\mathrm{ff}}\left(\lambda, T_{\mathrm{e}}\right) .
\end{aligned}
$$


Here $e$ denotes the electron charge, $c$ the speed of light, $\varepsilon_{0}$ the permittivity of free space, $m_{\mathrm{e}}$ the electron mass, $k_{\mathrm{B}}$ Boltzmann's constant, $\lambda$ the wavelength, $i$ the ionization stage and $N_{\mathrm{Ar}}$ the highest ionization stage to be considered. The so-called Biberman factors $\xi_{i}^{\mathrm{fb}}$ for free-bound and $\xi_{i}^{\mathrm{ff}}$ for free-free transitions of the electrons are expressed as one effective factor $\xi_{i}$ for each ionization stage $i$. For a singly ionized plasma $\left(N_{\mathrm{Ar}}=1\right.$, $n_{1}=n_{\mathrm{e}}$ ) the sum in Eq. (2) reduces to the first term $\left(n_{\mathrm{e}} \xi_{1}\right)$. This simplified version is used extensively in literature. For argon plasmas the simplification is a good approximation up to about $18000 \mathrm{~K}$. Above this temperature the inclusion of the next term $\left(4 n_{2} \xi_{2}\right)$ should be considered to avoid systematic errors as shown in [33].

In $[2,3]$ we chose the wavelength range between 4455 and $4456 \AA$ to measure the CEC, because this region does not contain argon line emission and $\xi_{1}$ is almost independent of both wavelength and temperature. Thus we assumed $\xi_{1}=1.86$, which is consistent with both theoretical [34] and experimental [35,36] data. The measured CEC is especially suitable to deduce the electron density $n_{\mathrm{e}}$, because $\varepsilon_{\lambda}$ depends mainly on $n_{\mathrm{e}}^{2}$, but only on $\sqrt{T_{\mathrm{e}}}$ and linearly on the $\xi$ factor. A detailed description of the simultaneous evaluation of line and continuum emission data in the pLTE case is given in [3].

We see large deviations from LTE near the cathode of our arcs [1,2] which are reminiscent of departures from LTE found in high-current arcs [7-11]. Even laser-scattering measurements showed discrepancies difficult to interpret, which have been resolved recently (see $[38,39]$ and references therein). For these devices discrepancies in the determination of the electron density are found in [9,37]. The authors of [10] suppose that this is caused by using the simplification of single ionization and neglecting the Ar III term $4 n_{2} \xi_{2}$ in Eq. (2). Therefore, we checked whether our results are influenced by a contribution of Ar III to the continuum emission.

Fig. 7 shows radially resolved values for $n_{\mathrm{e}}$ and $T_{\mathrm{e}}$, which we obtained in the first two sheaths in front of the cathode at a distance of 50 and $100 \mu \mathrm{m}$, respectively. To our knowledge the Biberman factor $\xi_{2}$ has not yet been measured for the wavelength $4455 \AA$ which we used for our measurements. The authors of [33] present measurements for $\xi_{2}$ around $4688 \AA$ for the temperature range $24000-28000 \mathrm{~K}$ and suggest a linear approximation for the temperature dependency. To check for the influence of $\xi_{2}$ in our measurements we assumed $\xi_{2}$ to be of similar magnitude for 4688 and $4455 \AA$, which is supported by calculations for $\xi_{2}$ in [36]. Furthermore, we extrapolated the linear approximation given in [33] to lower temperatures. This leads to a larger uncertainty for $\xi_{2}$, but should still be good enough to show the basic influence of $\xi_{2}$.

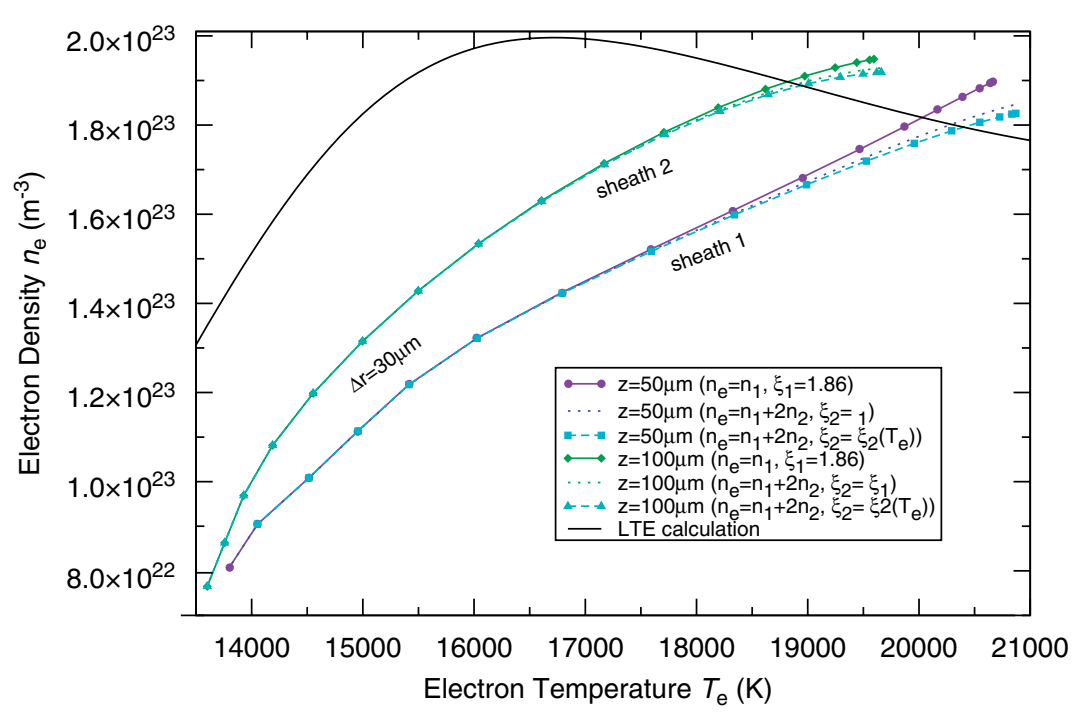

Fig. 7. Comparison of different methods to account for Ar III in continuum emission when calculating $n_{\mathrm{e}}$ and $T_{\mathrm{e}}$ from absolute LECs and CECs. The highest and lowest centre values of $\left(T_{\mathrm{e}}, n_{\mathrm{e}}\right)$ are $\left(2.087 \times 10^{4} \mathrm{~K}, 1.826 \times 10^{23} \mathrm{~m}^{-3}\right)$ and $\left(2.067 \times 10^{4} \mathrm{~K}, 1.898 \times 10^{23} \mathrm{~m}^{-3}\right)$ for sheath 1 , and $\left(1.966 \times 10^{4} \mathrm{~K}, 1.919 \times 10^{23} \mathrm{~m}^{-3}\right)$ and $\left(1.959 \times 10^{4} \mathrm{~K}, 1.948 \times 10^{23} \mathrm{~m}^{-3}\right)$ for sheath 2 . 
Thus we show three different curves for each sheath in Fig. 7 to get an overview of the influence of Ar III:

(1) Assuming a singly ionized plasma and using the simplified version of Eq. (2) $\left(n_{\mathrm{e}}=n_{1}, \xi_{1}=1.86\right)$ we obtain the highest electron densities and the lowest temperatures.

(2) Including the Ar III ionization stage and assuming $\xi_{2}$ to be equal to $\xi_{1}\left(n_{\mathrm{e}}=n_{1}+2 n_{2}, \xi_{1}=\xi_{2}=1.86\right)$ yields higher temperatures and lower electron densities. As expected the effect is largest in the centre of the arc.

(3) Including the Ar III ionization stage and using a linear extrapolation for the temperature dependency of $\xi_{2}$ based on the values from [33] and neglecting the wavelength dependency $\left(n_{\mathrm{e}}=n_{1}+2 n_{2}, \xi_{1}=1.86\right.$, $\left.\xi_{2}=\xi_{2}\left(T_{\mathrm{e}}\right)\right)$ increases $T_{\mathrm{e}}$ and lowers $n_{\mathrm{e}}$ only slightly.

From these curves we conclude that the influence of Ar III is noticeable and leads to a maximum systematic error of about $4 \%$ for $n_{\mathrm{e}}$ and $1 \%$ for $T_{\mathrm{e}}$ in the first sheath in front of the cathode where the highest values for $T_{\mathrm{e}}$ are reached. We can account for most of this error by including Ar III as highest ionization stage $n_{2}$ in the evaluation process. The actual value for the Biberman factor $\xi_{2}$ has only a small impact on the results: the curves for (2) and (3) in Fig. 7 are hardly distinguishable although their centre value for $\xi_{2}$ differs by about $20 \%$. Therefore, we expect to obtain reliable values for $n_{\mathrm{e}}$ and $T_{\mathrm{e}}$ using procedure (2) or (3). However, we note that for temperatures higher than in our arc (e.g. for the arc device used in [33]) the differences become larger and an exact value for $\xi_{2}$ is needed for the evaluation of the continuum emission to avoid systematic errors.

\section{Conclusion}

We have shown that some difficulties arise in quantitative spectroscopy on argon plasmas when taking Ar III into account. Although the method published by [15] to calculate partition functions is both convenient and accurate, the coefficients for Ar III published in [19] lead to questionable results. Unless the apparently incorrect coefficients are improved (preferably in the scope of a general update of [19] incorporating recent progress in the knowledge of atomic energy levels), a direct summation method should be used for Ar III instead. We encourage other authors to mention in their work how they calculate partition functions, because otherwise discrepancies like this cannot be traced back.

Motivated by the results published in [33] we checked for the influence of Ar III on the evaluation of continuum emission. We agree with the authors of [33] that Ar III should be included in the evaluation process at temperatures above $18000 \mathrm{~K}$. Therefore, the Biberman factor $\xi_{2}$ has to be known. For the temperature range of our arc the most important thing is to allow for Ar III in the first place, whereas the exact value for $\xi_{2}$ plays only a minor role. Nevertheless the contribution of Ar III becomes larger for higher temperatures, demanding reliable values of $\xi_{2}$. Thus measurements of $\xi_{2}$ over a larger temperature and wavelength region are desirable to extend the possibilities of continuum intensity diagnostics as a reliable and convenient method in plasma spectroscopy to higher temperatures.

Finally we note that the detailed analysis of the influence of Ar III hardly affects our previously published results, because in the temperature range in which our arc is operated, Ar III is still a minority species. Ar III can certainly not explain the large deviations from LTE, which can be seen in Fig. 7 and in $[1,2]$.

\section{Acknowledgment}

In memoriam of Hartmut Holweger. We thank K. Danzmann for admitting the completion of this work at the AEI, and M. Heurs for a critical reading of the manuscript.

\section{References}

[1] Reiche J, Könemann F, Mende W, Kock M. Diagnostics of discharge modes of a free-burning low-current argon arc. J Phys D: Appl Phys 2001;34:3177-84.

[2] Könemann F, Kühn G, Reiche J, Kock M. Near-cathode region of a free-burning arc: a spectroscopic investigation. J Phys D: Appl Phys 2004;37:171-9. 
[3] Kühn G, Kock M. A spatially resolved relaxation method for pLTE plasma diagnostics in free-burning arcs. J Phys D: Appl Phys 2006;39:2401-14.

[4] Könemann F. Emissionsspektroskopie im kathodennahen Bereich eines Lichtbogens, PhD Thesis, Hannover: Universität Hannover, http://edok01.tib.uni-hannover.de/edoks/e01dh01/3281269421.pdf (2000).

[5] Kühn G. Aktive und passive Plasmaspektroskopie im Kathodenbereich eines freibrennenden Lichtbogens. PhD thesis, Universität Hannover; Hannover. Berlin: Logos Verlag, ISBN 3-8325-1118-0; 2005.

[6] Pretzler G. A new method for numerical abel-inversion. Z Naturforsch 1991;46a:639-41.

[7] Pellerin S, Musiol K, Pokrzywka B, Chapelle J, Czernichowski A. Study of an electric arc in the vicinity of the cathode. J High Temp Chem Proc 1992;3(1):487-94.

[8] Pellerin S, Musiol K, Pokrzywka B, Chapelle J. Investigation of a cathode region of an electric arc. J Phys D: Appl Phys 1994;27:522-8.

[9] Razafinimanana M, Gudzy P, Gleizes A, El Hamidi L, Vaquié S. Experimental study of the cathode region of an argon transferred arc. High Temp Chem Proc 1995;4:51-8.

[10] Pokrzywka B, Musioł K, Pellerin S, Pawelec E, Chapelle J. Spectroscopic investigation of the equilibrium state in the electric arc cathode region. J Phys D: Appl Phys 1996;29:2644-9.

[11] Pokrzywka B, Musiol K, Pellerin S, Pawelec E, Cormier J-M, Chapelle J. Étude de la zone cathodique d'un arc électrique. C-R Acad Sci Paris Sér IIb 1999; t.327: 391-8.

[12] Olsen HN. Measurement of argon transition probabilities using the thermal arc plasma as a radiation source. JQSRT 1963;3:59-76.

[13] Olsen HN. The electric arc as a light source for quantitative spectroscopy. JQSRT 1963;3:305-33.

[14] Griem HR. High-density corrections in plasma spectroscopy. Phys Rev 1962;128(3):997-1003.

[15] Schlender B, Traving G. Tschebyscheff-Approximation von Zustandssummen. Z Phys 1965;61:92-4.

[16] Gündel H. Zustandssumme und effektive Ionisierungsspannung eines Atoms im Plasma und Möglichkeiten ihrer experimentellen Überprüfung. Teil I. Beitr Plasmaphys 1970;10:455-67.

[17] Gündel H. Zustandssumme und effektive Ionisierungsspannung eines Atoms im Plasma und Möglichkeiten ihrer experimentellen Überprüfung. II. Experiment. Beitr Plasmaphys 1971;11:1-12.

[18] Halenka J, Grabowski B. Remarks on atomic partition functions the example of tin. Astron Astrophys 1977;54:757-64.

[19] Traving G, Baschek B, Holweger H. Tabellen für die Berechnung von Zustandssummen. Abh Hamb Sternwarte 1966;VIII(1):3-25.

[20] Kurucz RL, Bell B. Atomic spectral line data base. Harvard Smithsonian Center for Astrophysics, vol. CDROM $23,1995$.

[21] Vlček J. A collisional-radiative model applicable to argon discharges over a wide range of conditions. I: formulation and basic data. J Phys D: Appl Phys 1989;22:623-31.

[22] Moore CE. Atomic energy levels, Circular of the National Bureau of Standards 467, vol. NSRDS-NBS 35, 1971.

[23] Lochte-Holtgreven W, editor. Plasma diagnostics. Amsterdam: North-Holland Publishing Company; 1968.

[24] Larenz RW. Über ein Verfahren zur Messung sehr hoher Temperaturen in nahezu durchlässigen Bogensäulen. Z Phys 1951;129(3):327-42.

[25] Kock M, Richter J. Der Einfluß statistischer Meßfehler auf die Lösung einer Abelschen Integralgleichung. Ann Phys Lpz 1969;24:30-7.

[26] Wiese WL, Brault JW, Danzmann K, Helbig V, Kock M. Unified set of atomic transition probabilities for neutral argon. Phys Rev A 1989;39(5):2461-71.

[27] Vujnović V, Wiese WL. A critical compilation of atomic transition probabilities for singly ionized argon. J Phys Chem Rev Data 1992;21(5):919-39.

[28] Pellerin S, Musiol K, Dzierzega K, Chapelle J. Measurement of atomic parameters of singly ionized argon lines-II. Transition probabilities. JQSRT 1997;57(3):359-76.

[29] Djeniže S, Bukvić S. Transition probabilities of several transtitions in the Ar III and Ar IV spectra. Astron Astrophys 2001;365:252-7.

[30] Kurucz RL. ATLAS: a computer program for calculating model stellar atmospheres. Smithsonian Astrophysical Observatory Special Report, vol. 309, 1970. p. 1-291.

[31] Wiese WL, Smith MW, Miles BM. Atomic transition probabilities vol. II. National Standard Reference Data Series, vol. NBS 22, 1969.

[32] Schulz-Gulde E. The continuous emission of argon in the visible spectral range. Z Phys 1970;230:449-59.

[33] Pokrzywka B, Pellerin S, Musiol K, Chapelle J. Experimental determination of the Ar III contribution to the continuum emission of argon. J Phys D: Appl Phys 1999;32:1665-70 [in French].

[34] Hofsaess D. Emission continua of rare gas plasmas. JQSRT 1978;19:339-52.

[35] Schnehage SE, Kock M, Schulz-Gulde E. The continuous emission of an argon arc. J Phys B: At Mol Phys 1982;15:1131-5.

[36] Wilbers ATM, Kroesen GMW, Timmermans CJ, Schram DC. The continuum emission of an arc plasma. JQSRT 1991;45(1):1-10.

[37] Gudzy P, Razafinimanana M, Gleizes A. Departures from equilibrium in the cathode region of an argon transferred arc at atmospheric pressure. Progress in plasma processing of materials - proceedings of the fourth international thermal plasma processes conference. New York: Begell House; 1997. p. 157-64.

[38] Murphy AB. Thomson scattering diagnostics of thermal plasmas: laser heating of electrons and the existence of local thermodynamic equilibrium. Phys Rev E 2004;69:016408.

[39] Dzierżęga K, Zawadzki W, Pokrzywka B, Pellerin S. Experimental investigations of plasma perturbation in Thomson scattering applied to thermal plasma diagnostics. Phys Rev E 2006;74:026404. 